19 Revue d'histoire du XIXe siècle

Société d'histoire de la révolution de 1848 et des

révolutions du XIXe siècle

$46 \mid 2013$

L'espace du politique en Allemagne au $\mathrm{XIX}^{\mathrm{e}}$ siècle

\title{
Eric Hobsbawm (1917-2012), un historien dans le siècle
}

Fabrice Bensimon

\section{OpenEdition}

\section{Journals}

Édition électronique

URL : http://journals.openedition.org/rh19/4457

DOI : $10.4000 /$ rh 19.4457

ISSN : 1777-5329

Éditeur

La Société de 1848

\section{Édition imprimée}

Date de publication : 1 juin 2013

Pagination : 185-190

ISSN : 1265-1354

\section{Référence électronique}

Fabrice Bensimon, «Eric Hobsbawm (1917-2012), un historien dans le siècle », Revue d'histoire du XIXe siècle [En ligne], 46 | 2013, mis en ligne le 01 juin 2016, consulté le 01 mai 2019. URL : http:// journals.openedition.org/rh19/4457 ; DOI : 10.4000/rh19.4457 


\section{ERIC HOBSBAWM (1917-2012), UN HISTORIEN DANS LE SIÈCLE ${ }^{1}$}

Eric Hobsbawm a souvent raconté sa vie, dont il disait qu'elle offrait un point de vue intéressant à l'historien qu'il était devenu². Il était né en 1917 à Alexandrie, dans l'Égypte sous protectorat britannique, de parents juifs. Son grand-père paternel polonais, un ébéniste, avait émigré en Grande-Bretagne dans les années 1870. Son père Leopold Percy Hobsbaum (changé en «Hobsbawm» par l'erreur d'un employé consulaire), un commerçant infortuné de l'East End londonien, avait rejoint son frère Sidney en Égypte. En 1913, il y rencontra Nelly Grün, une jeune Autrichienne de la classe moyenne, venue grâce à un prix scolaire. Le couple, issu de deux empires en guerre l'un contre l'autre, se maria en 1916 en Suisse neutre, retourna à Alexandrie où naquit Eric, avant de partir pour Vienne en 1919. Leopold mourut en 1929 d'une crise cardiaque, Nelly en 1931 de la tuberculose, et le jeune Eric, qui avait juste 14 ans, fut ensuite élevé par son oncle, qui vivait à Berlin. Eric Hobsbawm y adhéra au KPD, le Parti communiste allemand, et glissait encore des tracts sous les portes au cours des semaines qui suivirent l'accession au pouvoir d'Hitler. Avec ses frères et sœurs, il fut envoyé à Londres pour vivre avec la branche britannique de la famille.

Lycéen à la Marylebone Grammar School, il obtint ensuite une bourse pour étudier l'histoire à King's College, à Cambridge. Désormais membre du Parti communiste de Grande-Bretagne, Hobsbawm côtoya à Cambridge une génération d'intellectuels staliniens, dont les célèbres futurs espions, les "Cinq de Cambridge». Étudiant brillant, il se fit une réputation d'omniscience parmi ses condisciples. Il y étudia l'histoire, et fut marqué par Michael Postan, qui dirigeait l'Economic History Review à une époque où la discipline était encore surtout confinée à la politique et aux nations. Il passa ensuite sept années (1939-1946) sous les drapeaux, servant dans une unité de défense du territoire en East Anglia. Il disait que cette expérience l'avait "convertie» à la classe ouvrière britannique. Il fit à Cambridge une thèse sur les Fabiens (1947), mais n'y obtint pas le poste auquel ses compétences lui permettaient

1. Cette notice est parue en anglais, dans une version légèrement différente, dans la revue britannique Radical Philosophy, $\mathrm{n}^{\circ} 178$, mars-avril 2013. Lauteur remercie Laurent Colantonio, Joanna Innes, François Jarrige, Sheila Rowbotham et Julien Vincent pour leur relecture et leurs commentaires.

2. Interesting Times, 2002, traduction française : Franc-tireur. Autobiographie, Paris, Ramsey, 2005. 
de prétendre. Il obtint en 1947 un emploi de lecturer à Birkbeck (université de Londres), un ancien Mechanics' Institute devenu college et spécialisé dans les cours pour adultes. Hobsbawm avait coutume de dire qu'il menait ses recherches pendant la journée et dispensait ses cours le soir. Il y devint professeur en 1970 et y enseigna jusqu’à la retraite en 1982. Il anima longtemps le séminaire de "Modern British History" de l'université de Londres, au sein de l'Institute of Historical Research. Il devint ainsi un vrai Londonien, demeurant à Hampstead avec sa femme Marlene, qu'il avait épousée en secondes noces en 1962, et avec qui il entretenait une riche vie sociale. Eric Hobsbawm avait déjà un fils, Joss, et eut deux autres enfants avec Marlene, Andy et Julia.

À la fin des années 1940, Hobsbawm avait adhéré au Communist Party Historians' Group, aux côtés de Christopher Hill, Rodney Hilton, E. P. Thompson, George Rudé, John Saville, Victor Kiernan ou Leslie Morton. C'est ce groupe qui fonda, en 1952, la revue Past and Present, qui fut soustitrée A Journal of Scientific History jusqu'en 1958, quand elle s'ouvrit à des historiens non-marxistes, comme Moses Finley ou Lawrence Stone. Inspirée par l'école française des Annales avec laquelle elle partageait l'intérêt pour les études sur la longue durée, Past and Present s'intéressait à l'histoire sociale et économique et à l'histoire internationale - alors que l'Angleterre restait le terrain privilégié des historiens anglais. Past and Present voulait publier des articles tout à la fois fondés sur une recherche originale et accessible aux non-spécialistes. Hobsbawm resta toute sa vie associé à la revue. Et considéra toujours que l'histoire était une sorte de science, s'opposant ainsi au «tournant linguistique» des années 1980.

Ses livres étaient marqués par cette empathie avec les opprimés et cet intérêt pour l'histoire par en bas (bistory from below), qu'il a gardés toute sa vie. Un de ses premiers articles, paru dans le numéro un de Past and Present, était consacré aux briseurs de machines ${ }^{3}$. Un de ses premiers ouvrages, Primitive Rebels (1959) $)^{4}$, portait sur les sociétés secrètes rurales de l'époque moderne, ceux que Hobsbawm appelait les "bandits sociaux", précédant les formes organisées de mouvement social; il prolongea cette recherche dans Bandits (1969)5. Labour's Turning point (un recueil de sources paru en 1948), Labouring Men (1964) et Worlds of Labour (1984) traitent de différents aspects de la classe ouvrière britannique, avec un intérêt particulier pour la période 1880-1920, quand, écrivait-il, la classe ouvrière s'est réinventée autour des syndicats du new unionism, des coopératives, du socialisme et du Labour, mais aussi de la casquette, du football, du cinéma et du fish and chips. Captain Swing (1969), une étude systématique qu'il mena avec George Rudé, est

3. "The machine breakers", Past and Present, $\mathrm{n}^{\circ}$ 1, février 1952, pp. 57-70.

4. Les Primitifs de la révolte dans l'Europe moderne, Paris, Fayard, "L'Histoire sans frontières», 1963.

5. Les Bandits, Paris, François Maspéro, 1972. 
longtemps demeurée la seule monographie disponible sur les émeutes rurales du Sud de l'Angleterre en 1830, un objet par définition difficile à saisir.

Le champ favori d'Eric Hobsbawm était l'histoire économique et sociale, et son nom est étroitement associé à cet âge d'or qu'elle connut en GrandeBretagne au cours des années 1960 à 1980. Un de ses chefs-d'œuvre en la matière fut Industry and Empire (1968), une histoire économique de la Grande-Bretagne depuis 1750, qui intégrait dans un même mouvement la première révolution industrielle et la constitution du plus grand empire. Hobsbawm fut un des protagonistes du célèbre débat sur les niveaux de vie (standard of living debate) pendant l'industrialisation, critiquant ceux qui n'y voyaient qu'un progrès de la condition ouvrière. "C'est une bonne époque pour être un historien du social» ${ }^{6}$, écrivait Hobsbawm en 1971, quand l'histoire sociale avait conquis sa place dans le champ universitaire, avec ses réseaux, ses colloques et ses revues, et avait en quelque sorte ringardisé l'histoire politique. Il est difficile de choisir quel article ou quelle thèse évoquer, tant ses intérêts étaient divers. Une de ses contributions les plus originales fut peut-être celle sur les ouvriers itinérants (tramping artisans) de l'industrialisation, terrassiers, chaudronniers et autres mécaniciens (in Labouring Men, 1964). Hobsbawm n’a guère pratiqué la micro-histoire, ni la biographie. En revanche, c'était un historien du social, dans toutes ses dimensions, si on excepte peut-être l'histoire des femmes, assez peu présente dans ses travaux. Bien avant que l'histoire culturelle n'acquière la place qu'elle a aujourd'hui, Hobsbawm s'intéressait aux pratiques culturelles, aux jeux et aux sports, aux loisirs et à la musique. Sous le pseudonyme de Francis Newton, il a publié de nombreux articles sur le jazz, une autre de ses passions (The Jazz Scene, 1959; Uncommon People: Resistance, Rebellion and Jazz, 1998) ${ }^{7}$. Surtout, il intégrait la culture à tous ses livres, à ses grandes synthèses notamment, qui réservaient toujours des chapitres consacrés aux arts et aux sciences, aux religions et aux courants de pensée. Comme l'ont dit nombre de ceux qui l'ont côtoyé, il s'intéressait à la littérature, à la musique classique, à tout en fait; voire, comme le rapportait Tony Judt, «il savait tout».

C'est sa tétralogie qui lui valut la réputation internationale qui est aujourd'hui la sienne. The Age of Revolution: 1789-1848 (1962), The Age of Capital: 1848-1875 (1975), The Age of Empire: 1875-1914 (1987) et The Age of Extremes: 1914-1991 (1994) sont devenus des incontournables des listes de lecture universitaires en Grande-Bretagne puis, au fil des dizaines de traductions, dans le monde ${ }^{8}$. Euvres d'histoire globale à une époque où

6. "It is a good time to be a social historian", in Eric. J. Hobsbawm, "From social history to the history of society", Daedalus, nº 100,1971 , p. 20-45.

7. Rébellions - La résistance des gens ordinaires : jazz, paysans et prolétaires, traduction de Stéphane Ginsburgh et Hélène Hiessler, Bruxelles, Éditions Aden, 2010.

8. En traduction française : L'Ére des révolutions: 1789-1848, Paris, Fayard, 1970; L'Ère du capital : 1848-1875, Paris, Fayard, 1978; L'Ére des empires : 1875-1914, Paris, Fayard, 1989; L'Âge des extrêmes : le court XXe siècle 1914-1991, Paris-Bruxelles, Le Monde diplomatique - Éditions Complexe, 1999. 
l'essentiel de la production historique avait pour cadre les structures nationales, ces quatre volumes restent marqués par des qualités qui font consensus : un exceptionnel sens de la synthèse; un goût prononcé pour l'histoire comparée; un sens aigu du quantitatif; et en même temps un usage pertinent de l'anecdote. Cette série fut d'abord conçue comme une trilogie sur la période qui va des "deux révolutions» (française et industrielle) à la Première Guerre mondiale. Bien qu'il ait écrit aussi bien sur le $\mathrm{XX}^{\mathrm{e}}$ que sur le XVII siècle, Hobsbawm disait que "son" siècle était ce "long XIX ${ }^{e}$ siècle", ainsi qu'il l'appelait avec une expression qui a fait florès. Même le plus ancien des volumes, L'Ére des révolutions, demeure, cinquante ans après sa parution, une des meilleures introductions à la période. L'Âge des extrêmes est venu ensuite et ce dernier volume porte sans doute, plus que les précédents, la trace des errements politiques de Hobsbawm, à propos d'une période dont il était un témoin engagé autant que l'historien. Cette tétralogie était fondée sur une vaste bibliographie que seul pouvait maitriser un polyglotte comme lui, qui, outre l'anglais et l'allemand, parlait couramment le français, l'italien et l'espagnol, et lisait le portugais et le catalan. Le style de Hobsbawm, dépourvu de tout jargon, ne laisse pas d'impressionner par sa précision et sa clarté. "Les historiens ne doivent pas écrire seulement pour d'autres historiens", écrivait-il ${ }^{9}$. Ces quatre volumes ont peut-être fait d'Eric Hobsbawm l'historien le plus connu au monde. On retrouve sa maitrise de l'histoire mondiale dans une réflexion sur la construction des identités nationales, Nations and Nationalism since $1780(1991)^{10}$, une question qu'il avait également abordée dans un ouvrage co-dirigé avec Terence Ranger, The Invention of Tradition (1983) ${ }^{11}$, et dont la thèse continue d'être embrassée par tous ceux qui étudient les traditions. Sa renommée a largement dépassé les frontières européennes : aux États-Unis (il enseigna de 1984 à 1997 à la New School for Social Research à New York), en Inde, en Chine, en Amérique latine, au Brésil en particulier, il a été largement traduit, lu et invité. Il faut dire qu'Eric Hobsbawm était, comme il disait lui-même, un vrai «cosmopolite», à même de débattre aux quatre coins de la planète. Il était critiqué aussi, comme il se doit. Par exemple, alors que Hobsbawm est très étudié en Inde, Ranajit Guha lui reprochait d'avoir sous-estimé le caractère politique des luttes paysannes. Edward Saïd jugeait sa tétralogie trop centrée sur l'Europe et le monde occidental.

Mais c'est surtout sa profession de foi «communiste» qui lui valait de solides inimitiés. Parmi les conservateurs, d'abord; lors de son décès, l'historien A. N. Wilson l'a ainsi accusé, dans une notice aux relents de guerre froide, d'être un «menteur» dont on ne lirait plus les livres à l'avenir, de

9. Franc-Tireur. Autobiographie, p. 339.

10. Nations et nationalismes depuis 1780 : programmes, mythe et réalité, Paris, Gallimard, 1992.

11. L'Invention de la tradition, Paris, Éditions Amsterdam, 2006. 
«haïr la Grande-Bretagne», voire d'avoir été un "traittre " ${ }^{12}$. Mais les critiques venaient aussi d'intellectuels de la gauche social-démocrate, comme Tony Judt $^{13}$. Hobsbawm épousa tous les zigzags du stalinisme. À la différence de nombreux intellectuels britanniques (dont E.P. Thompson, Christopher Hill ou Raphael Samuel), il ne quitta pas le Parti communiste, ni en 1956, lors de l'écrasement de la révolution hongroise par l'armée soviétique, ni plus tard. S'il émit des critiques du stalinisme, elles étaient en quelque sorte bienveillantes. Il n'avait plus d'activité militante mais, à la différence d'intellectuels français comme François Furet ou Annie Kriegel, il ne fut pas un repenti du communisme. Même quand il disait s'être trompé sur le stalinisme, il ne renonçait pas au marxisme, dont il se réclama jusquau bout (How to Change the World: Tales of Marx and Marxism, 2011) ${ }^{14}$. "J'appartiens à la génération pour qui la révolution d'Octobre représentait l'espoir du monde», écrivaitil ${ }^{15}$. Il expliquait souvent que son attachement au communisme était une fidélité à son engagement de jeunesse, dans les derniers mois de la République de Weimar. En même temps, à partir de la fin des années 1970, il enjoignit le parti travailliste de changer pour s'adapter aux évolutions sociologiques de l'électorat (le supposé déclin de la classe ouvrière), pour s'adresser plus directement à la middle class, ferraillant ainsi contre la gauche travailliste ${ }^{16}$. Hobsbawm acquit une posture d' 'intellectuel de gauche», plutôt modéré en fait ${ }^{17}$. Son marxisme était une méthode d'analyse du passé et du présent, bien plus qu'un outil pour changer le monde. Il était très largement reconnu, bien au-delà des historiens marxistes, au-delà de la gauche même. Le dirigeant travailliste Neil Kinnock disait de lui qu'il était son «marxiste préféré», et, en 1998, Tony Blair fit Hobsbawm membre de l'Ordre des compagnons d'honneur - un nombre très limité de personnalités récompensées pour services rendus à la nation. Il avait acquis non seulement une grande notoriété mais aussi une certaine respectabilité au sein de l'intelligentsia britannique, voire européenne. Et lors de son décès, même l'historien néo-conservateur Niall Ferguson a dressé son éloge, au nom de leur intérêt partagé pour l'histoire économique et de leur amitié.

Hobsbawm connaissait bien la France, où il se rendait presque chaque année depuis les années 1930. Il disait avoir commencé à apprendre le français auprès de sa mère, à une époque où «c'était toujours la seconde langue universelle». Dans son autobiographie, il y consacre tout un chapitre («Marseillaise»); une photo le montre à Paris le 14 juillet 1936, sur une voiture de

12. A. N. Wilson, "He hated Britain and excused Stalin's genocide. But was hero of the BBC and the Guardian, Eric Hobsbawm a traitor too?", Daily Mail, 2 octobre 2012.

13. Tony Judt, "The last romantic», New York Review of Books, 20 novembre 2003.

14. Voir par exemple : Marx et l'histoire, textes inédits traduits par Christophe Magny, Paris, Demopolis, 2008

15. Franc-tireur, op. cit., p. 77.

16. "The Forward March of Labour Halted", Marxism Today, 1978.

17. Politics for a rational Left: political writing, 1977-1988, Londres, Verso, 1989. 
la SFIO, et une autre en 1937 lors d'une conférence étudiante de soutien à la République espagnole. Il raconte que lorsqu'il était à Cambridge, 'Mounia' Postan avait invité Marc Bloch en le présentant comme le plus grand médiéviste de son temps. Hobsbawm lisait ce qui paraissait en France et s'était lié, écrivait-il, à des intellectuels français comme Henri Raymond et Hélène Berghauer, Richard et Élise Marienstras, Fernand Braudel, Ernest Labrousse ou Pierre Bourdieu. Il écrivit dans des revues comme Le Monde diplomatique. Ses ouvrages d'histoire européenne ou globale étaient traduits. Il ferraillait contre les interprétations révisionnistes d'Alfred Cobban puis de François Furet, spécialistes de la Révolution française (Echoes of the Marseillaise : Two Centuries Look Back on the French Revolution, 1990) ${ }^{18}$. Au milieu des années 1990, une polémique eut lieu à propos de The Age of Extremes (1994): l'ouvrage eut beau être traduit dans de nombreuses langues, aucun éditeur ne voulut publier une version française, ce que l'historien Pierre Nora, de Gallimard, justifia en disant qu'il fallait «tenir compte de la conjoncture intellectuelle et idéologique», autrement dit, que les idées de Hobsbawm avaient fait leur temps, avec la chute du mur de Berlin. Le Monde diplomatique et l'éditeur belge Complexe publièrent une traduction, qui rencontra un vaste succès. Et Hobsbawm est resté, jusqu'au bout, invité par les universités et les médias français, le plus connu sans doute des historiens étrangers.

$*$

Il est toujours difficile de prévoir ce qui restera d'un auteur. Hobsbawm n'a pas fondé d'école et a eu, en fin de compte, peu de disciples à proprement parler. Comme toute œuvre historique, la sienne est vouée à être dépassée. Mais plusieurs de ses livres sont si divers dans leurs objets, si riches d'érudition et parfois si généreux dans leur intérêt pour les oubliés de l'histoire, qu'il est à gager que notre époque n'a pas fini de lire avec profit, avec sens critique aussi, cet historien indissociable du «court $\mathrm{XX}^{\mathrm{e}}$ siècle».

Fabrice Bensimon

18. Aux armes, historiens. Deux siècles d'histoire de la Révolution française, postface inédite de l'auteur, traduit de l'anglais par Julien Louvrier, Paris, La Découverte, 2007. 